\title{
The Syntax of the Abstract-type Measurement Construction in Mandarin Chinese
}

Jing Jin

The Education University of Hong Kong

\begin{abstract}
This paper investigates a special sub-type of measurement construction in Mandarin Chinese, namely the [Num-measure word- $d e-\mathrm{N}$ ] construction where the $\mathrm{N}$ is an abstract dimension-denoting noun. Evidence is presented to show that the abstract-type [Num-measure word- $d e-\mathrm{N}$ ] should be fundamentally distinguished from the quantifying-/modifying-type [Num-measure word-de-N], in which the [Num-measure word] sequence serves to quantize/modify a semantically concrete, entity-denoting N. At the interpretive level, this paper claims that the abstracttype [Num-measure word-de-N] is semantically definite. At the syntactic level, a clausal analysis within the framework of the Predicate Inversion theory is pursued to account for the derivation of the abstract-type measurement construction. Last, it is proposed that the word order distinction between the Chinese abstracttype measurement construction, which is N-final, and its English counterpart, where the $\mathrm{N}$ linearly precedes [Num-measure word], can be explained in terms of a parametric variation with respect to the (non-)application of $\mathrm{N}$-raising after Predicate Inversion.
\end{abstract}

\section{Keywords}

Measurement construction, predicate inversion, word order, Chinese syntax

Studies in Chinese Linguistics, Volume 38, Number 1, 2017, 35-61 DOI: 10.1515/scl-2017-0003 (C2017 by T.T. Ng Chinese Language Research Centre, Institute of Chinese Studies, The Chinese University of Hong Kong 


\section{Introduction}

The formation of measure expressions in Mandarin Chinese has been a subject of immense scholarly interest in recent years. Chinese measure words (MW henceforth), such as jin 'catty', bang 'pound', mi 'meter', li 'mile', sheng 'liter', etc., can participate in forming two measurement constructions, i.e. [Num-MW-N] and [Num-MW-de-N]. It is well-noted that whereas [Num-MW] in [Num-MW-N] consistently conveys a quantificational meaning, as shown in (1a), [Num-MW] in [Num-MW-de-N] may serve as either a quantifier or a modifier, as illustrated in (1b) and (1c), respectively (Hsieh 2008; Jiang 2008; Her \& Hsieh 2010; X. Li 2011; Jin 2013; Y.-H. Li 2013; Zhang 2013).

(1) a. liang sheng shui

two liter water

'two liters of water'

b. liang sheng de shui

two liter DE water

'two liters of water'

c. liang sheng de shuihu

two liter DE pot

'a two-liter pot / two-liter pots'

As regards Chinese $d e$-contained measurement constructions, it has been shown in many studies that the quantifying-type [Num-MW-de-N] and the modifyingtype [Num-MW-de-N], albeit identical in linear word order, exhibit asymmetric syntactic behaviors. To illustrate, the two behave differently in (i) the ellipsis of the $\mathrm{N}$, (ii) the accommodation of a preceding quantifier, and (iii) the omission of $d e$, as demonstrated in (2)-(4). To capture this, various formal analyses have been attempted in previous studies to assign distinct underlying structures to the two constructions (Jiang 2008; Hsieh 2008; X. Li 2011; Jin 2013; Y.-H. Li 2013; Zhang 2013).

(2) (Non-)) licensing of $\mathrm{N}$-ellipsis

a. Lisi he le liang sheng de shui,

Lisi drink Perf two liter DE water

wo he le yi sheng de *(shui).

I drink Perf one liter DE water

(Quantifying-type)

Intended: 'Lisi drank two liters of water; I drank one liter (of water).'

b.Lisi mai de shi liang sheng de shuihu,

Lisi buy DE be two liter DE pot

wo mai de shi yi sheng de (shuihu).

I buy $\mathrm{DE}$ be one liter $\mathrm{DE}$ pot

(Modifying-type)

'What Lisi bought is a two-liter pot / are two-liter pots; what I bought is a two-liter one / are two-liter ones.' 
(3)(Non-)licensing of a preceding quantifier

a. *henduo liang sheng de shui a.lot.of two liter DE water

'*a lot of two liters of water'

b. henduo liang sheng de shuihu

a.lot.of two liter DE pot

'a lot of two-liter pots'

(4)(Non-)licensing of de-omission

a. liang sheng (de) shui

two liter DE water

'two liters of water'

b. liang sheng $*($ de $)$ shuihu

two liter DE pot

(Quantifying-type)

(Modifying-type)

Intended: 'a two-liter pot / two-liter pots'

(Quantifying-type)

(Modifying-type)

The present paper discusses another sub-type of [Num-MW-de-N] which has not drawn much scholarly attention in the literature. This type of [Num-MW-de-N] is most obviously different from the much discussed quantifying-/modifying-type [Num-MW-de-N] in that while the $\mathrm{N}$ contained in the latter is associated with concrete entities/substance, the $\mathrm{N}$ in the former denotes abstract dimension categories such as rongji 'volume', changdu 'length', and zhongliang 'weight', as shown in (5):

(5) a.liang sheng de rongji

two liter DE volume

'the volume of two liters'

b.wu mi de changdu

five meter DE length

'the length of five meters'

c.shi bang de zhongliang

ten pound DE weight

'the weight of ten pounds'

For ease of exposition, [Num-MW-de-N] as shown in (5) will be called the abstracttype [Num-MW-de-N] henceforth. This paper is organized as follows. Section 2 conducts a comparison between the abstract-type [Num-MW-de-N] and the quantifying-/modifying-type [Num-MW-de-N]. Section 3 explores the semantic and syntactic differences between the abstract-type [Num-MW-de-N] and [NumMW]. Section 4 proposes a formal analysis to account for the derivation of the abstract-type [Num-MW-de-N], followed by discussion on the consequences of this analysis in Section 5. Section 6 concludes this paper.

\section{Characteristic properties of the abstract-type [Num-MW-de-N]}

To obtain a preliminary picture of the characteristics of the abstract-type [Num$\mathrm{MW}-d e-\mathrm{N}]$, it is imperative to compare this construction to the quantifying-/ 
modifying-type [Num-MW-de-N] first. Upon a close examination, the following distinctions are detected.

To begin with, the abstract-type [Num-MW-de-N] differs from the quantifyingtype [Num-MW-de-N] in that while de contained in the latter is optional, de in the former is obligatory, as illustrated by the contrast below:

(6) a. liang sheng (de) shui (=(4a))

two liter DE water

'two liters of water'

b. wu mi (de) bu

five meter DE cloth

'five meters of cloth'

(7) a. liang sheng *(de) rongji

two liter DE volume

'the volume of two liters'

b. wu mi *(de) changdu

five meter DE length

'the length of five meters'

In the meanwhile, the abstract-type [Num-MW-de-N] behaves differently from the modifying-type [Num-MW-de-N] in the following respects. First, while the modifying-type [Num-MW-de-N] allows the $\mathrm{N}$ to be elided, this is by no means permitted in the case with the abstract-type [Num-MW-de-N]:

(8)a. wo xiang mai shuihu, ni juede

I want buy pot you think

liang sheng de (shuihu) gou da ma?

two liter DE pot enough big SFP

'I want to buy a pot / pots. Do you think the two-liter one / two-liter ones would be big enough?'

b. wo xiang mai rongji da yidian de shuihu,

I want buy volume big a.little DE pot

zuihao neng you liang sheng de *(rongji).

better can have two liter DE volume

Intended: 'I want to buy a pot / pots with a little larger volume, which better can reach the volume of two liters.'

Second, while the modifying-type [Num-MW-de-N] can be preceded by another quantifier, the abstract-type [Num-MW-de-N] cannot:

\begin{tabular}{|c|c|c|c|c|}
\hline ) a. henduo & liang & sheng & de & shuihu \\
\hline lot.of & two & liter & $\mathrm{DE}$ & pot \\
\hline $\begin{array}{l}\text { b. *henduo } \\
\text { a.lot.of }\end{array}$ & $\begin{array}{l}\text { liang } \\
\text { two }\end{array}$ & $\begin{array}{l}\text { sheng } \\
\text { liter }\end{array}$ & $\begin{array}{l}\text { de } \\
\text { DE }\end{array}$ & $\begin{array}{l}\text { rongji } \\
\text { volume }\end{array}$ \\
\hline
\end{tabular}


Third, [Num-MW-de] in the modifying-type [Num-MW-de-N] can be stacked with another $d e$-marked modifier to give rise to a parallel modification construction, which is nevertheless not allowed for the [Num-MW-de] sequence in the abstracttype [Num-MW-de-N], as illustrated below:

(10)a. liang sheng de, yong le henduo nian de shuihu two liter DE use Perf many year DE pot 'the pot which is two liters and which has been used for many years'

b. *liang sheng de, gang ce chulai de rongji two liter DE just measure out DE volume 'the volume which is two liters and which was just measured out'

It is worth indicating that the above shown characteristics of the abstract-type [Num-MW-de-N] cannot be simply attributed to the abstract nature of the $\mathrm{N}$ contained. To understand this point, compare the abstract-type [Num-MW-de-N] to the ordinary Chinese de-marked modification constructions composed by dimension-denoting head nouns, as given in (11):

(11) a. zhe jian fangzi de mianji

this $\mathrm{Cl}$ room $\mathrm{DE}$ area

'the area of this room'

b. gang ce chulai de changdu

just measure out DE length

'the length which was just measured out'

c. chaobiao de tizhong

exceed-standard DE weight

'the weight which exceeds the standard'

What have been shown in (11) are all typical de-marked modification constructions. Intriguingly, albeit the Ns contained also denote abstract dimension categories, the examples in (11) do not syntactically pattern with the abstract-type [Num$\mathrm{MW}-d e-\mathrm{N}]$ in a number of ways. To be specific, the examples in (11) allow the $\mathrm{N}$ to be elided (cf. (12a)), can accommodate a preceding quantifier (cf. (12b)), and are compatible with parallel modification (cf. (12c)), all of which, as has been illustrated earlier, are not permitted for the abstract-type [Num-MW-de-N]. This indicates that the characteristics of the abstract-type [Num-MW-de-N] as exhibited in (8)-(10) should not be attributed to the abstract lexical meaning of the $\mathrm{N}$ involved.

(12) a. shuodao mianji, zhe jian fangzi de (mianji) zui xiao. speaking.of area this $\mathrm{Cl}$ room $\mathrm{DE}$ area most small 'Speaking of area, that of this room is the smallest.'

b. henduo gang ce chulai de changdu dou you wenti. a.lot.of just measure out DE length all have problem 'A lot of lengths which were just measured out are problematic.' 


\section{c. chaobiao de, hui yingxiang shenti jiankang de tizhong} exceed-standard DE will affect body health DE weight 'the weight which exceeds the standard and which will affect people's health'

To summarize, this section showed that the abstract-type and the quantifying-/ modifying-type [Num-MW-de-N], although apparently parallel in linear word order, exhibit asymmetric syntactic behaviors. Also, it was demonstrated that it is inappropriate to consider the abstract-type [Num-MW-de-N] as falling under the de-marked modification construction in Mandarin Chinese. ${ }^{1}$

\section{The abstract-type [Num-MW-de-N] and [Num-MW]}

\subsection{Semantic differences}

To explore the very nature of the abstract-type [Num-MW-de-N], it helps to first look into the role played by the dimension-denoting $\mathrm{N}$ and the semantic differences between the [Num-MW] phrase and the abstract-type [Num-MW-de-N]. In approaching this, the core idea to be pursued in this paper is that "measure units" and "dimension categories" are two different notions. This can be best illustrated by the lack of a strict one-to-one correspondence between measure words and dimension categories. That is, it is possible for a measure word to be associated with various dimension categories depending on the context or the speaker's intension. Taking (13), observe that the measure word $m i$ 'meter' is associated with the dimension "height" in (a), a two-way dimension ambiguity between "length" and "width" in (b), a three-way dimension ambiguity concerning "length", "width", and "height" in (c), and a three-way dimension ambiguity among "perimeter", "diameter", and "radius" in (d). ${ }^{2}$

1 Interested readers are referred to Jin (2016) for a syntactic investigation into the quantifying-type and the modifying-type [Num-MW-de-N], which are assumed as underlyingly correlating with the DP-internal Focus Phrase and the Modifier Phrase, respectively, with de as a DP-internal focus marker in the former and as a modification marker in the latter.

2 At this point, one may propose that the dimension ambiguity exhibited by the measure word $m i$ 'meter' as in (13) can be disambiguated via the use of an adjective immediately following [Num$\mathrm{MW}$ ]. Compare (i) to (13c) for example. The use of chang 'long', kuan 'wide', and gao 'tall' right after the [Num-MW] sequence explicitly indicates that $m i$ is intended to be associated with the dimension "length", "width", and "height", respectively.

(i) liang $\mathrm{mi}$ chang/kuan/gao de guizi

two meter long/wide/tall DE closet

'a two-meter long/wide/tall closet; two-meter long/wide/tall closets'

Nevertheless, it is worth noticing that the use of adjectives right after [Num-MW] cannot apply in all cases. For instance, there are no adjectives in Chinese dedicated to dimensions such as perimeter, diameter, and radius; therefore, (13d) can by no means appropriately have a [NumMW-Adj-de-N] counterpart as (13c) does. Besides, there are measure words which generally resist being accompanied by adjectives, including those concerning electric current (e.g. an 'ampere'), voltage (e.g. $f u$ ' 'volt'), and so on. For [Num-MW] sequences composed by these measure words, as shown in (ii) below, no adjectives can be appropriately used to indicate the intended dimension categories. 
(13) a. liang mi de yundongyuan

two meter DE player

'a two-meter (tall) player / two-meter (tall) players'

b. liang mi de guodao

two meter DE aisle

'a two-meter (long/wide) aisle / two-meter (long/wide) aisles'

(ii) a. 16 an de chazuo

16 ampere DE socket

'a 16-ampere socket / 16-ampere sockets'

b. $220 \mathrm{fu}$ de dianqi

220 volt DE appliance

'a 220-volt appliance / 220-volt appliances'

One reviewer raises a further question as to how to explain the expressions as follows:

(iii) san $\mathrm{mi}$ chang de changdu

three meter ong DE length

The present paper suggests that the [Num-MW-Adj] sequence as shown in (iii) be accounted for along the line Schwarzschild (2005) and Moltmann (2009) propose for two meters long in English. In accordance with their analysis, the [Num-MW] phrase (e.g. two meters) preceding a gradable adjective (e.g. long) is a degree modifier. More in specific, the pre-adjective measure phrase is treated essentially akin to an adverbial such as very, upon the analysis that both of them semantically serve to provide specifying information concerning the degree of the attribute denoted by the following adjective. Along this approach, two meters long is considered structurally identical to very long.

In the same vein, this paper assumes that san mi chang should be associated with the same underlying structure as that of hen chang ('very long'), and it is considered that for both san mi chang de changdu and hen chang de changdu, the head noun changdu 'length' is modified by a degree adjective phrase (i.e., san mi chang 'three meters long' in the former and hen chang 'very long' in the latter), with the whole construction meaning 'a/the length which is three meters long' and ' $a$ /the length which is very long', respectively. Empirical evidence in support of the stance that [Num-MW-Adj-de-N] should be analyzed as a de-marked modification construction can be found from the fact that the [Num-MW-Adj-de] sequence, just like [Adv-Adj-de], can participate in forming parallel modification constructions, a typical characteristic of de-marked modifiers (cf. Section 2). In this respect, [Num-MW-Adj-de] behaves differently from the [Num-MW-de] sequence contained in the abstract-type [Num-MW-de-N] as the latter is not compatible with parallel modification (cf. Section 2). Consider the following examples:

(iv) a. hen chang de, yuanyuan chaoguo yuanding biaozhun de changdu verylong DE far exceed original standard DE length 'the length which is very long and which far exceeds the original standard'

b. san $\mathrm{mi}$ chang de, yuanyuan chaoguo yuanding biaozhun de changdu three meterlong DE far exceed original standard DE length 'the length which is three meters long and which far exceeds the original standard'

c. *san mi de, yuanyuan chaoguo yuanding biaozhun de changdu three meter DE far exceed original standard DE length

With this at hand, it can be seen clearly that it is necessary to distinguish the abstract-type [Num$\mathrm{MW}-d e-\mathrm{N}]$ from [Num-MW-Adj-de-N]. I thank the reviewer for bringing examples like (iii) into my attention. 
c. liang mi de guizi two meter DE closet

'a two-meter (long/wide/tall) closet / two-meter (long/wide/tall) closets

d. liang $\mathrm{mi}$ de yuanquan

two meter DE circle

'a circle / circles with two meters in perimeter/diameter/radius'

In view of this, this paper proposes that the abstract-type [Num-MW-de-N] construction is an expression formed to explicitly convey complete information concerning (i) the measure result, which is denoted by [Num-MW], and (ii) the associated dimension category, which is specified by the N. Taking (14) to demonstrate the distinction in information completeness of the abstract-type [Num-MW-de-N] as compared to [Num-MW], observe that the reply (a), which adopts [Num-MW], is pragmatically odd, whereas the reply (b), which uses the [Num-MW-de-N] construction, is well-accepted. This shows that [Num-MW] in itself is not sufficient to provide full information on the size of the plate concerned in the given context. ${ }^{3}$

(14) ni dui panzi de daxiao you shenme yaoqiu? you about plate DE size have what requirement

'Do you have any requirements on the size of the plate?'

a. \#zhishao yao you 20 limi.

at.least should have 20 centimeter

'\#It should at least reach 20 centimeters.'

b. zhishao yao you 20 limi de zhijing/banjing.

at.least should have 20 centimeter DE diameter/radius

'It should at least reach the diameter/radius of 20 centimeters.'

In conclusion, measure units cannot be treated on a par with dimension categories. It is the abstract-type [Num-MW-de-N] that provides full information concerning the measure value on the one hand and the associated dimension on the other hand, while the [Num-MW] phrase in itself merely denotes a measure value.

3 A scenario that might allow for either [Num-MW-de-N] or [Num-MW] without resulting in inappropriateness is when the dimension category concerned is the pre-established knowledge mutually shared by the interlocutors. In that case, even though one simply utters [Num-MW], as the intended dimension category is contextually salient, no pragmatic oddity would arise, as shown in the following:

(i) ni dui panzi de zhijing/banjing you shenme yaoqiu?

you about plate DE diameter/radius have what requirement

'Do you have any requirements on the diameter/radius of the plate?'

zhishao yao you 20 limi.

at.least should have 20 centimeter

'It should at least reach 20 centimeters.' 


\subsection{Syntactic differences}

In addition to the semantic distinctions, it is observed that the abstract-type [Num-MW-de-N] and [Num-MW] also exhibit different syntactic behaviors. To be specific, first, [Num-MW] can be well used as a predicative expression whereas [Num-MW-de-N] cannot. This can be illustrated by (15): regardless of how appropriate the contextual motivation is, the abstract-type [Num-MW-de-N], in contrast with [Num-MW], cannot be directly adopted as a reply to how-type questions, which ask for predicative/property-denoting answers.

(15) a. zhe ge guodao duo kuan?

this $\mathrm{Cl}$ aisle how wide

'How wide is this aisle?'

liang mi. / \#liang mi de kuandu.

two meter two meter DE width

'(\#The width of) two meters.'

b. zhe liang che kai de duo kuai?

this $\mathrm{Cl}$ car drive $\mathrm{DE}$ how fast

'How fast is the car running?'

80 mai. /\#80 mai de sudu.

80 mile-per-hour 80 mile-per-hour DE speed

'(\#The speed of) 80 miles per hour.'

Second, while [Num-MW] can be used to quantize noun denotations, this is not allowed for [Num-MW-de-N]. As shown in (16), [Num-MW] can be juxtaposed with an entity-denoting noun to form a quantifying construction, whereas [Num$\mathrm{MW}-d e-\mathrm{N}]$ can never be used this way.

(16) a. liang sheng shui / *liang sheng de rongji shui two liter water two liter $\mathrm{DE}$ volume water Intended: 'two liters of water'

b. wu mi bu $/{ }^{*}$ wu mi de changdu bu five meter cloth five meter DE length cloth Intended: 'five meters of cloth'

c. qi bang putao $/ *^{\text {qi }}$ bang de zhongliang putao seven pound grape seven pound DE weight grape Intended: 'seven pounds of grapes'

All this taken together, this paper claims that the abstract-type [Num-MW-de-N], notwithstanding the involvement of a semantically highly abstract head noun, cannot be taken equivalent to [Num-MW] in use. The two should be distinguished at a fundamental level. 


\section{A clausal analysis for the abstract-type [Num-MW-de-N]}

\subsection{A closer look at the abstract-type [Num-MW-de-N]}

Before getting down to a formal analysis, a closer look will be taken at the semantics of the abstract-type [Num-MW-de-N] and the correlation between [Num-MW] and the dimension-denoting $\mathrm{N}$ therein.

As regards the very semantic nature of the abstract-type [Num-MW-de-N], the stance to be taken in this paper is that this construction conveys a definite meaning. Specifically, the abstract-type [Num-MW-de-N] is semantically akin to a proper name in that it stands on its own to refer to a particular measure value along a welldefined dimension category. Due to this property, the contexts where one may utter this expression are generally those in which the main discourse concern/interest lies in a dimension-determined measure value alone rather than in concrete entities/ substance with this measure value, as exemplified by the bracketed argumental [Num-MW-de-N] expressions given as follows:

(17)a. [liang mi de kuandu] chengyi [san mi de changdu] two meter DE width multiply three meter DE length dedao [liu pingfangmi de mianji]. obtain six square-meter DE area

'The width of two meters multiplied by the length of three meters gives the area of six square meters.'

b. [liang mi de kuangdu] dagai jiu shi tingchechang li two meter DE width probably just be parking-lot in yi tai che de kuandu. ${ }^{4}$

one $\mathrm{Cl}$ car $\mathrm{DE}$ width

'The width of two meters is just (equivalent to) the width of a car in the parking lot.'

c. [50 pingfangmi de mianji] wei ni tigong le yi ge 50 square-meter DE area for you provide Perf one CL kuanchang de woshi he dandu de xiuxiqu. ${ }^{5}$ spacious DE bedroom and separate DE resting-area 'The area of 50 square meters provides with you a specious bedroom and a separate resting area.'

A further piece of evidence for the definite, proper name-like nature of the abstract-type [Num-MW-de-N] comes from the fact that this phrase, parallel to ordinary proper names, can allow for topicalization and can serve as the topic in the topic-comment construction, as illustrated below: ${ }^{6}$

4 Example from: http://mypaper.pchome.com.tw/tokai/post/1321510520.

5 Example from: http://www.hoteldux.nl/cn/hotel-rooms/suite-deluxe.

6 I thank one reviewer for pointing out this diagnostics for definiteness to me. The examples in (18) are from the reviewer. 
(18) a. Zhangsan, wo zuotian jian guo.

Zhangsan I yesterday see Exp

'Zhangsan, I saw (him) yesterday.'

b. Zhangsan, wo zhidao ta xihuan Lisi.

Zhangsan, I know he like Lisi

'(As for) Zhangsan, I know he likes Lisi.'

(19) a. liang mi de zhijing, wo juede hen chang. two meter DE diameter I think very long

'The diameter of two meters, I think it is long.'

b. liang mi de zhijing, wo juede zhe ge chicun tai da. two meter DE diameter I think this $\mathrm{Cl}$ size too big '(As for) the diameter of two meters, I think this size is too big.'

The definite semantics of the abstract-type [Num-MW-de-N] can be best manifested by the formation of its counterpart in English. To express a particular measure value along a certain dimension, English adopts the form of [the-Nof-Num-MW], with definiteness explicitly encoded by the determiner the, as exemplified below:

(20) a. The radius of 63 meters was measured out accurately. ${ }^{7}$

b. However, ligers in reality can reach the weight of nearly 1200 pounds. ${ }^{8}$

c. Finally, kilometer and myriameter shall be the lengths of 1,000 and 10,000 meters, and shall designate principally the distances of roads. ${ }^{9}$

Now turn to the semantic relationship between the constituents within the abstracttype [Num-MW-de-N]. The core claim to be made in this paper is that the [Num$\mathrm{MW}$ ] sequence and the dimension-denoting $\mathrm{N}$ contained in this construction entertain a predication relationship underlyingly. To spell out this idea, notice that [Num-MW] can always be directly predicated of the dimension-denoting noun in the copular sentence, as shown in (21). This contrasts with the case with the entity-denoting noun. As illustrated in (22), unless the information concerning the intended dimension category has been given/presupposed in the context, generally an entity-denoting noun cannot be directly predicated of by $[\mathrm{Num}-\mathrm{MW}]$ in the copular sentence.

(21) a. kuandu shi liang mi.

width be two meter

'The width is two meters.'

b. mianji shi 150 pingfangmi.

area be 150 square-meter

The area is 150 square meters.'

7 Example from: http://www.bibliotecapleyades.net/ciencia/antigravityworldgrid/ciencia antigravityworldgrid08.htm.

8 Example from: http://www.ligerworld.com/weighing-liger-vs-tiger-vs-lion.html.

9 Example from: https://chnm.gmu.edu/revolution/d/463/. 
c. dianya shi 220 fu.

voltage be 220 volt

'The voltage is 220 volt.'

(22) a. yundongyuan \#(de shengao) shi liang mi.

player DE height be two meter

'\#(The height of) the player is two meters.'

b. guodao \#(de changdu) shi liang mi. aisle DE length be two meter

'\#(The length of) the aisle is two meters.'

c. guizi \#(de kuandu) shi liang mi.

closet DE width be two meter

'\#(The width of) the closet is two meters.'

Further evidence supporting the stance that measure value-concerned expressions can be directly predicated of dimension-denoting nouns but not of entity-denoting nouns comes from the fact that the question word duoshao 'how much', which asks for a measure result, can directly apply to dimension-denoting nouns but not to entity-denoting nouns to form copular sentences, as illustrated in (23) and (24):

(23) a. kuandu shi duoshao?

width be how.much

'How much is the width?'

b. mianji shi duoshao?

area be how.much

'How much is the area?'

c. dianya shi duoshao?

voltage be how.much

'How much is the voltage?'

(24) a. yundongyuan \#(de shengao) shi duoshao?

player DE height be how.much

'How much is \#(the height of) the player?'

b. guodao \#(de changdu) shi duoshao?

aisle DE length be how.much

'How much is \#(the length of) the aisle?'

c. guizi \#(de kuandu) shi duoshao?

closet DE width be how.much

'How much is \#(the width of) the closet?'

To recapitulate, so far, two claims have been made concerning the semantic properties of the abstract-type [Num-MW-de-N]. First, the construction as a whole, which refers to a particular measure value along a well-defined dimension, is semantically definite. Second, at the interpretive level, there is a predication relationship between the [Num-MW] phrase and the N contained, with [NumMW], which specifies the measure value, being predicated of the dimensiondenoting N. Based on this, the following subsection develops a formal analysis 
for the abstract-type [Num-MW-de-N] within the framework of the Predicate Inversion theory.

\subsection{Derivation of the abstract-type [Num-MW-de-N]}

Upon the claim that in terms of semantics the [Num-MW] sequence and the dimension-denoting $\mathrm{N}$ entertain a predication relationship, this paper attempts to account for the derivation of the abstract-type [Num-MW-de-N] under the DP-internal Predicate Inversion theory (Bennis et al. 1998; den Dikken \& Singhapreecha 2004; den Dikken 2006, 2007). Within this theoretical framework, it is assumed that the dimension-denoting $\mathrm{N}$ and the [Num-MW] sequence start out forming a small clause in the underlying structure, with [Num-MW] the predicate of the N. Following the terminology of den Dikken $(2006,2007)$, it is hypothesized that at the structural level, the subject and the predicate of the small clause is connected via the functional head RELATOR, with the small clause correlating with the RELATOR-headed functional projection called RP, as visualized below:

(25) Step 1:

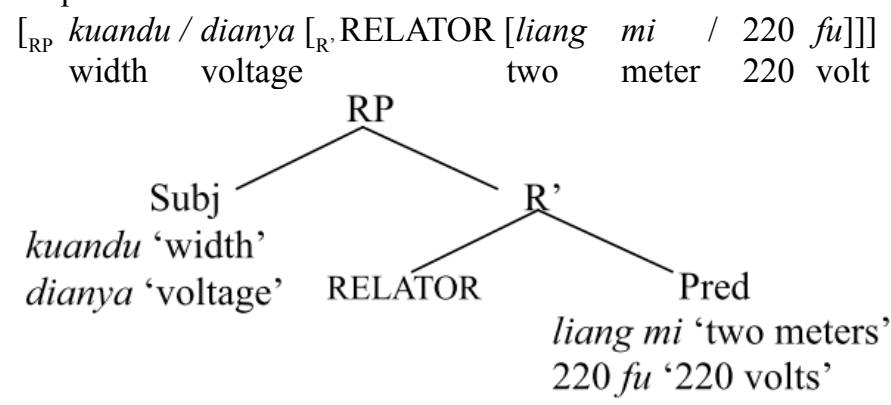

To obtain the right word order, it is postulated that the operation of Predicate Inversion will apply to the structure (25). That is, the predicate of the RP, that is, liang $\mathrm{mi} / 220 \mathrm{fu}$, moves across the subject kuandu / dianya to the specifier of a higher functional projection on top of the RP. The functional head whose specifier provides the landing site for the inverted predicate is LINKER (also following the terminology of den Dikken); the functional projection headed by LINKER is labeled LP in this paper for the sake of expository simplicity. Importantly, to ensure the well-formedness of the resultant structure, two requirements are assumed for Predicate Inversion. First, RELATOR has to move to LINKER to render [Spec, LP] and [Spec, RP] equidistant with respect to the underlying predicate, an instance of phase extension in the sense of Chomsky (2000). Second, the LINKER head, whose specifier hosts the inverted predicate, needs to be overtly realized (Bennis et al. 1998; den Dikken \& Singhapreecha 2004; den Dikken 2006, 2007). As far as the structure (25) is concerned, it is assumed that the inversion of [Num-MW] across the dimension-denoting $\mathrm{N}$ is legitimated by the RELATOR-to-LINKER movement and accompanied by the phonological realization of LINKER as de, as 
visualized below: $:^{10}$

(26) Step 2:

$\left[_{\mathrm{LP}}[\text { liang } m i / 220 f u]_{\mathrm{j}}\left[{ }_{\mathrm{L}}, \operatorname{LINKER}+\operatorname{RELATOR}_{\mathrm{i}}(=d e)\left[_{\mathrm{RP}}\right.\right.\right.$ kuandu / dianya $\left.\left.\left.\left[_{\mathrm{R}}, \mathrm{t}_{\mathrm{i}} \mathrm{t}_{\mathrm{j}}\right]\right]\right]\right]$

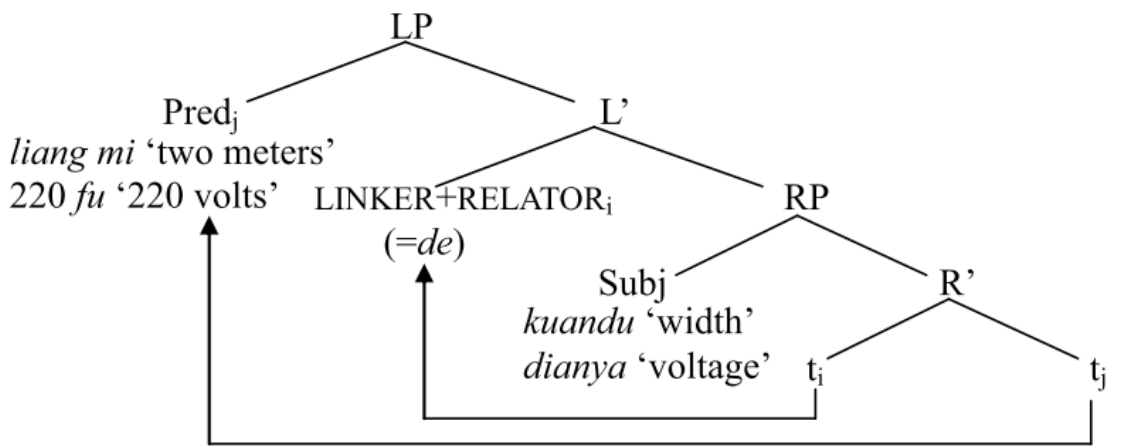

Last, given that the abstract-type [Num-MW-de-N] is semantically definite (cf. Section 4.2), upon a standard assumption that referentiality is encoded in the functional head D (Longobardi 1994; Chomsky 2000; Zamparelli 2000; Chierchia 2005), it is hypothesized that the structure (26) will continue projecting till the DP layer. In accordance with the licensing condition on functional projections

10 One reviewer asks a question as to what is the motivation of the inversion of liang $m i$ / $220 \mathrm{fu}$ as assumed here. This paper considers that the movement of the predicate liang $m i / 220 \mathrm{fu}$ from the complement of R to [Spec, LP] is fundamentally motivated by the need to convert a small clause RP into a nominal expression via breaking the original structural relationship between the subject and the predicate, namely, via altering the relative word order between the subject and the predicate. As such, Predication Inversion is taken as a grammatical means to turn clausal constructions into derived complex nominal constructions. This spirit has been widely exercised in the literature to deal with empirical data within the Predicate Inversion theory. Taking Dutch for instance, the bracketed phrases as given below, albeit quite different in semantics, are both analyzed as starting out as clauses and ending up as nominal expressions because of the application of Predicate Inversion (Bennis et al. 1998):

(i) a. [een beer van een vent]

a bear of a man

(from Bennis et al. (1998): (2a))

b. [Wat een boeken] heb jij gelezen?

what a books have you read

'Boy, did you read a lot/kind of books!'

(from Bennis et al. (1998): (35c))

There is a rather theory-internal explanation attempted in den Dikken (2006) regarding the motivation of Predicate Inversion within the nominal domain (which is though just mentioned briefly without much elaboration in his book). The author postulates that all nominal phrases deriving from Predicate Inversion underlyingly involve a small clause whose predicate is headed by an empty head (den Dikken 2006: 180). Based on this, he attributes the obligatory Predicate Inversion inside the nominal domain to the need to formally license the empty predicate head. As the technical analysis for the obligatoriness of Predicate Inversion is not the focus of the present study, this paper does not delve into this issue. Interested readers are referred to den Dikken (2006) for detailed discussion. 
as proposed in Dimitrova-Vulchanova and Giusti (1998) and Zamparrelli (2000), which claims that the projection of a functional layer needs to be licensed by the lexical realization of (at least) the specifier or the head of the functional layer, it is assumed that upon the projection of the DP, [Num-MW] will undergo raising from [Spec, LP] to [Spec, DP], as depicted in (27). Upon completion of this step, the right surface word order and the desired semantics of the abstract-type [Num$\mathrm{MW}-d e-\mathrm{N}]$ is finally obtained. ${ }^{11}$

11 One reviewer raises an intriguing question regarding the derivation of Step 3, that is, how to account for the co-occurrence of the demonstrative $z h e$ 'this' or $n a$ 'that' with the abstract-type [Num-MW-de-N] if the latter correlates with DP in the underlying structure:

(i) zhe/na liangmi de kuandubu suanshenme.

this/that twometer DE width not countwhat

'This/That width of two meters is nothing (at all).'

To explain example (i), it is worth noticing that this sentence is most naturally uttered when some entity identifiable to both the speaker and the hearer - which is two meters in width is contextually given and salient for the current conversation. Specifically, suppose there is a contextually identified road which speaker A considers wide whereas speaker B considers narrow. Supposing speaker A says "The road is really wide", it is appropriate for speaker B to adopt sentence (i) as a reply to express his/her disagreement to A's statement (probably accompanied by a pointing gesture to the road under discussion). Notice that in this case, the demonstrative is used deictically, serving to refer to the width associated with the road contextually identified to the interlocutors. However, by contrast, if a demonstrative is not used deictically, the resultant [Dem-Num-MW-de-N] would lead to inappropriateness. Consider the following conversation:

(ii) A: women xiang xiu lu, liang $\mathrm{mi}$ de kuandu fuhe biaozhun ma? we want build road two meter DE width satisfy standard SFP

'We want to build a road. Would the width of two meters meet the standard?'

B: *zhe/na liang mi de kuandu yinggai bu xing. this/that two meter DE width should not work

'*This/That width of two meters should not work.'

For (ii), as speaker A and speaker B are talking about a future plan of building roads, no roads have been brought into existence at the moment the conversation takes place. Given this, [DemNum-MW-de-N] in this case, instead of being deictically associated with a contextually identified referent, can only be understood as anaphorically referring to the preceding [Num-MW-de-N] expression. As indicated by the asterisk, the demonstrative irrelevant to the deictic usage is not allowed to co-occur with the abstract-type [Num-MW-de-N].

In view of the picture shown here, the present paper intends to approach examples like (i) under the Split DP analysis developed in Alexiadou et al. (2007: 127-129). Under this analysis, the DP field can be further split into an articulated array of functional projections, that is, while the discourse information such as deixis is encoded in the higher layer of DP (i.e. DP), the determinationrelated meaning, such as definiteness and indefiniteness, is conveyed by the lower layer of DP (i.e. $\mathrm{DP}_{2}$ ). Along this line, the demonstrative in the [Dem-Num-MW-de-N] sequence, which exhibits a deictic reading, should correlate with the higher $\mathrm{DP}_{1}$; while the abstract-type [Num-MW-de-N], being semantically definite yet lacking a deictic interpretation in itself, should correlate with the lower $\mathrm{DP}_{2}$, as visualized below. As such, the co-occurrence of the demonstrative and the abstracttype [Num-MW-de-N] in (i) can receive a straightforward explanation.

(iii) $\left[_{\mathrm{DP} 1 \text { (deixis) }} z h e / n a\right.$... [ ${ }_{\mathrm{DP} 2 \text { (determination) }}$ liang mi de kuandu $\left.]\right]$ 
(27) Step 3:

$\left[_{\mathrm{DP}}[\text { liang } m i / 220 f u]_{\mathrm{j}} \mathrm{D}\left[_{\mathrm{LP}} \mathrm{t}_{\mathrm{j}}\left[_{\mathrm{L}} \mathrm{LINKER}_{\mathrm{R}}+\mathrm{RELATOR}_{\mathrm{i}}(=d e)\left[_{\mathrm{RP}} k u a n d u /\right.\right.\right.\right.$ dianya $\left.\left.\left.\left.\left[{ }_{R}, \mathrm{t}_{\mathrm{i}} \mathrm{t}_{\mathrm{j}}\right]\right]\right]\right]\right]$

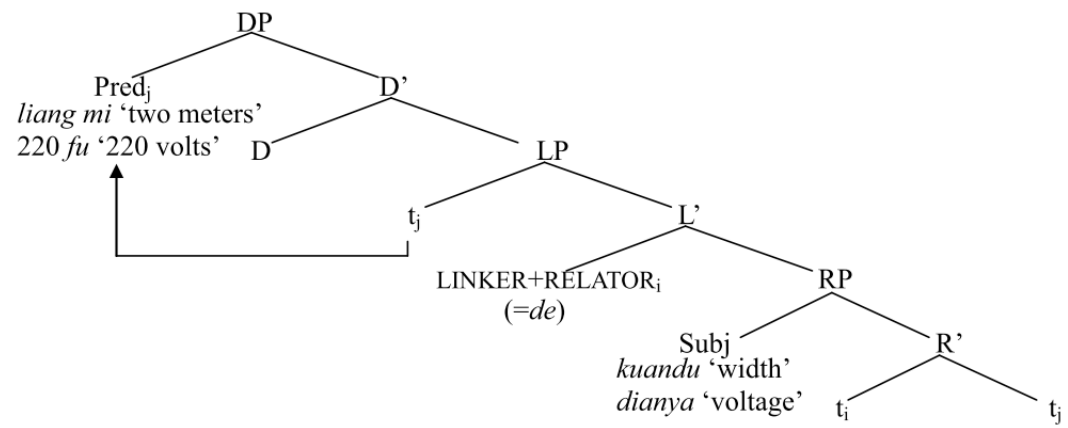

Confronted with such a clausal analysis, one might immediately raise a question as to how this line could deal with the word order exhibited by the English counterpart of the Chinese abstract-type [Num-MW-de-N], which is in the form of [the-N-of-Num-MW] as has been seen in (20):

(28) a. the radius of 63 meters

b. the weight of nearly 1200 pounds

c. the lengths of 1,000 and 10,000 meters

Concerning this, this paper proposes that the observed word order difference between English and Chinese is due to a parametric variation with respect to the derivational process languages undergo in forming abstract-type measurement constructions. The core hypothesis is that, while abstract-type measurement constructions in both Chinese and English start the derivation with a small clause structure and involve Predicate Inversion in the course of derivation, discrepancy emerges between them as to whether the remnant subject left by Predicate Inversion will undergo further raising.

To be concrete, this paper assumes that the first two derivational steps undergone by the English abstract-type measurement construction are exactly the same as those of the Chinese abstract-type measurement construction, as shown in (29). The LINKER head, whose specifier provides the landing site for the inverted predicate, is spelled out as of in English. 
(29) a. Step 1:

${ }_{\mathrm{RP}}$ radius/weight ${ }_{\mathrm{R}}$, RELATOR [63 meters / nearly 1200 pounds $\left.]\right]$

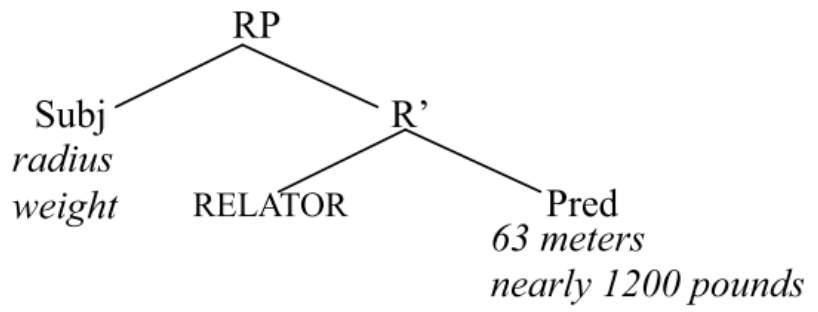

b. Step 2:

$\left[_{\mathrm{LP}}[63 \text { meters/nearly } 1200 \text { pounds }]_{\mathrm{j}}\left[_{\mathrm{L}}\right.\right.$, LINKER+RELATOR ${ }_{\mathrm{i}}(=$ of $)\left[_{\mathrm{RP}}\right.$ radius/weight $\left.\left.\left.\left[{ }_{\mathrm{Rel}}, \mathrm{t}_{\mathrm{i}} \mathrm{t}_{\mathrm{j}}\right]\right]\right]\right]$

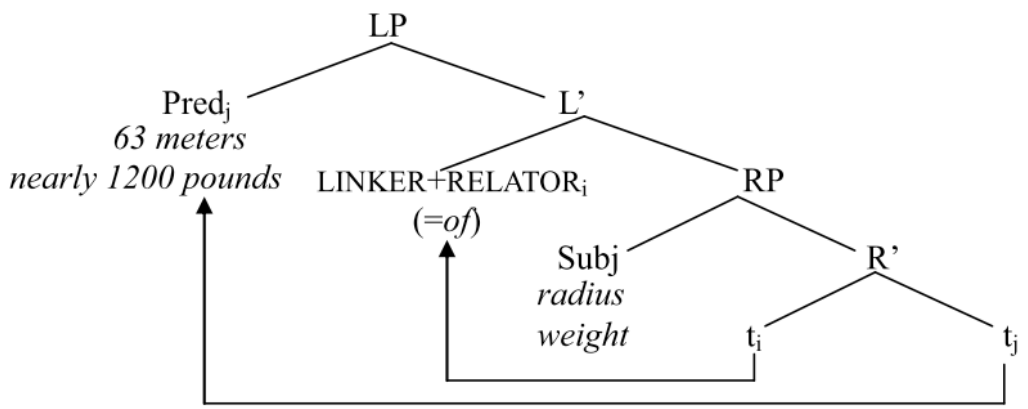

Crucially, this paper assumes that upon completion of Step 2, the remnant subject, i.e. radius/weight at [Spec, RP], will undergo movement into a higher functional layer FP immediately on top of LP. The remnant movement will undo the wordorder effect brought about by Predicate Inversion and restore the $\mathrm{N}$-initial word order, as visualized in (30). As the movement of radius/weight from [Spec, RP] to [Spec, FP] involves skipping of an intermediate specifier position (i.e. [Spec, LP]), to make this movement legitimate, the head complex LINKER+RELATOR formed in Step 2 needs to raise to the F head to render [Spec, FP] and [Spec, LP] equidistant with respect to radius/weight in [Spec, RP]. 
(30) Step 3:

$\left[_{\mathrm{FP}}[\text { radius/weight }]_{1}\left[_{\mathrm{F}}, \mathrm{F}+\left[\text { LINKER }+\operatorname{RELATOR}_{\mathrm{i}}(=o f)\right]_{\mathrm{k}}\left[_{\mathrm{LP}}[63\right.\right.\right.$ meters $/$ nearly 1200 pounds $\left.\left.\left.]_{\mathrm{j}}\left[\mathrm{L}_{\mathrm{L}}, \mathrm{t}_{\mathrm{k}}\left[{ }_{\mathrm{RP}} \mathrm{t}_{\mathrm{i}}\left[\mathrm{L}_{\mathrm{R}}, \mathrm{t}_{\mathrm{i}} \mathrm{t}_{\mathrm{j}}\right]\right]\right]\right]\right]\right]$

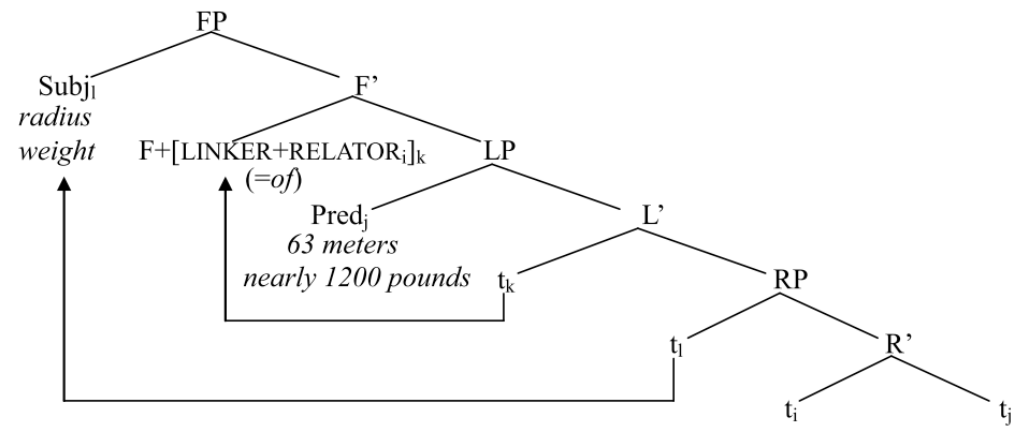

Last, the structure obtained after Step 3 projects into the DP level. Upon insertion of the determiner the under $\mathrm{D}$, the configuration of [the-N-of-Num-MW] is obtained.

(31) Step 4:

$\left[_{\mathrm{DP}}\right.$ the $\left[_{\mathrm{FP}}[\text { radius } / \text { weight }]_{1}\left[_{\mathrm{F}}, \mathrm{F}+\left[\text { LINKER }+ \text { RELATOR }{ }_{\mathrm{i}}(=o f)\right]_{\mathrm{k}}\left[_{\mathrm{LP}}[63\right.\right.\right.$ meters $/$ nearly 1200 pounds $\left.\left.\left.\left.]_{j}\left[{ }_{L}, t_{k}\left[{ }_{R P} t_{1}\left[{ }_{R}, t_{i} t_{j}\right]\right]\right]\right]\right]\right]\right]$

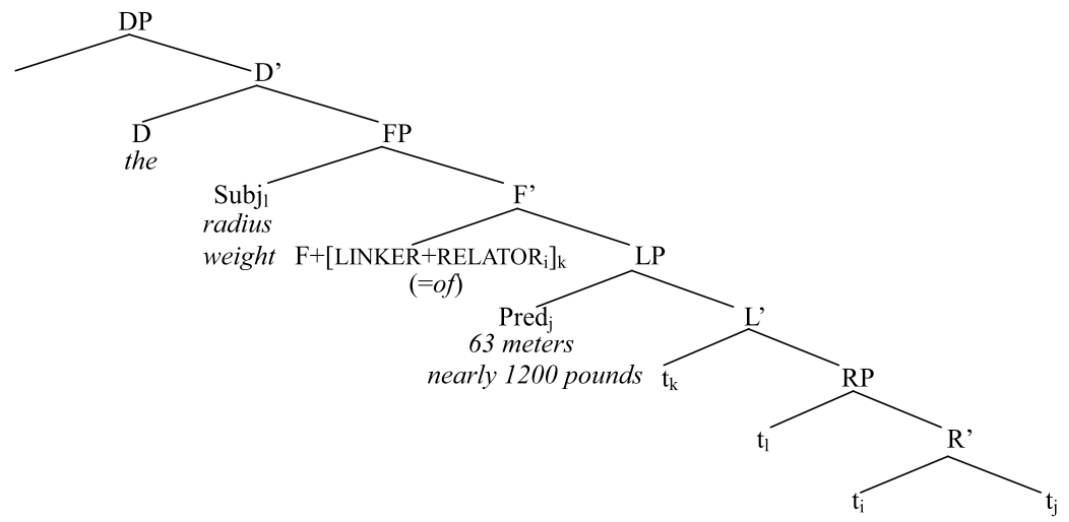

To sum up, this section proposed a clausal analysis for the derivation of the abstracttype [Num-MW-de-N]. Based on the fact that in terms of interpretation the dimension-denoting $\mathrm{N}$ and [Num-MW] stand in a direct predication relationship, it is assumed that underlyingly the two start with a clausal structure in the form of [N-[Num-MW]]. The surface word order of [Num-MW-de-N] is a result of syntactic derivation involving the application of Predicate Inversion, upon which the predicate [Num-MW] is fronted across the subject $\mathrm{N}$ and de spells out the functional head whose specifier hosts the inverted predicate. Last, an explanation was provided as to why the English counterpart of the Chinese abstract-type [Num-MW-de-N] exhibits a different word order, i.e. [the-N-of-Num-MW]. The present analysis attributed this word order distinction to a parametric variation as 
to whether Predicate Inversion is followed by remnant subject movement, which has an effect of restoring the N-comes-first word order in the surface structure.

\subsection{An alternative}

The present Predicate Inversion approach may remind one of Kayne's (2003) analysis for the age of eleven years/months, a construction that appears quite similar to the abstract-type measurement construction [the-N-of-Num-MW] in English. In fact, the age of eleven years/months can be well thought of as falling under the abstract-type measurement construction if one views "age" as a kind of dimension category and the entire construction, concomitantly, as an ageconcerned measurement construction.

As regards the derivation of the age of eleven years/months, Kayne hypothesizes that age and eleven years/months entertain a predication relationship in the underlying structure. Nevertheless, somehow different from the proposal presented above, Kayne treats the dimension-denoting $\mathrm{N}$ age as the underlying predicate while the measure result eleven years/months as the underlying subject, which is opposite to the treatment of the present paper. Consider (32) for illustration (irrelevant details omitted for expository simplicity):

$$
\begin{aligned}
& {\left[_ { \mathrm { DP } } \text { the } \left[_ { \mathrm { LP } } \text { age } { } _ { \mathrm { j } } \mathrm { L } _ { \mathrm { L } } \text { LINKER+RELATOR } ( = o f ) \left[_ { \mathrm { RP } } \text { eleven years/months } \left[_{\mathrm{R}}, \mathrm{t}_{\mathrm{i}}\right.\right.\right.\right.} \\
& \left.\left.\left.\left.\mathrm{t}_{\mathrm{j}}\right]\right]\right]\right]^{12}
\end{aligned}
$$

While this approach seems to be a tempting alternative to the proposal offered here, the present paper does not intend to follow it because of the following considerations. First, empirical evidence turns out to be more compatible with the analysis of [Num-MW] as the predicate of the dimension-denoting $\mathrm{N}$ rather than the other way around. As manifested by the contrast below, while the copular sentence which has the dimension-denoting $\mathrm{N}$ as the subject and [Num-MW] as the predicate can be appropriately formed, that containing [Num-MW] as the subject and the dimension-denoting $\mathrm{N}$ as the predicate is decidedly odd:

(33) $[N u m-M W]$ as the predicate

a.kuandu shi liang mi.

width be two meter

'The width is two meters.'

b.mianji shi 150 pingfangmi.

area be 150 square-meter

'The area is 150 square meters.'

c. dianya shi 220 fu.

voltage be 220 volt

'The voltage is 220 volts.' $\quad(=(21))$

12 The labels RELATOR/RP and LINKER/LP are not adopted in Kayne's work but used here for consistency with the terminology used in Section 4.2. 
(34) Dimension-denoting $N$ as the predicate
a.??liang mi shi kuandu.
two meter be width
b.??150 pingfangmi shi mianji.
150 square-meter be area
c. ??220 fu shi dianya.
220 volt be voltage

Second, on the theoretical side, notice that if applying the line as demonstrated in (32) to the Chinese abstract-type measurement constructions (namely holding that the underlying small clause of the Chinese abstract-type measurement construction is [[Num-MW]-N]), then external stipulations would be called for to explain the [Num-MW-de-N] word order exhibited by the Chinese abstract-type measurement construction (in contrast with [the-N-of-Num-MW] in English). This issue, nevertheless, can be well dealt with under the present analysis. Recall from Section 4.2 that the word order difference between English and Chinese abstracttype measurement constructions is due to a parameter with respect to the (non-) application of remnant subject movement after Predicate Inversion. Notice that this is not an ad hoc solution only applicable to abstract-type measurement constructions. In fact, the same idea has been pursued in the literature to explain the distinction between Chinese and other languages (e.g. French, English) in terms of word order of possessive and partitive constructions (den Dikken \& Singhapreecha 2004; den Dikken 2007; Jin 2015). Taking the derivation of possessives for illustration, den Dikken and Singhapreecha (2004) and den Dikken (2007) have claimed that although possessive constructions universally start with a small clause in the form of "Possessum-Possessor", languages may vary in the particular derivational process they undergo to derive the surface structure. Concretely, while the derivation of Chinese possessives involves Predicate Inversion only, the derivation of French possessives involves further raising of the remnant subject after Predicate Inversion. As a result, Chinese possessives end up with the surface word order of [Possessor$d e$-Possessum], whereas French possessives exhibit the order of [Possessum-dePossessor] $]{ }^{13}$ See the visualization of the derivation below:

$$
\begin{aligned}
& \text { Chinese Possessives: Predicate Inversion Only }
\end{aligned}
$$

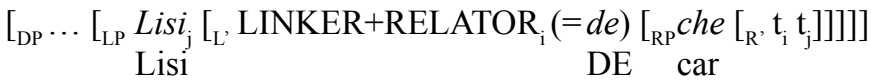

(36) French Possessives: Predicate Inversion plus Remnant Subject Raising

a. Step 1: Predicate Inversion

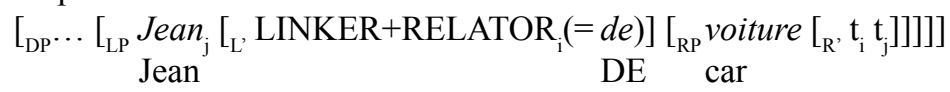

13 The same spirit has been followed by Jin (2015) in accounting for the word order distinction between Chinese-type and English-type partitive constructions. Interested readers are referred to this paper for detailed discussion. 
b. Step 2: Remnant Subject Raising

$\left[_{\mathrm{DP}} \cdots\left[_{\mathrm{FP}}\right.\right.$ voiture $_{1}\left[_{\mathrm{F}}, \mathrm{F}+\left[\mathrm{LINKER}+\mathrm{RELATOR} \mathrm{i}_{\mathrm{i}}(=d e)\right]_{\mathrm{k}}\left[_{\mathrm{LP}}\right.\right.$ Jean $_{\mathrm{j}}\left[_{\mathrm{L}}, \mathrm{t}_{\mathrm{k}}\left[_{\mathrm{RP}} \mathrm{t}_{\mathrm{l}}\left[_{\mathrm{R}}, \mathrm{t}_{\mathrm{i}}\right.\right.\right.$ $\mathrm{t}_{\mathrm{j}}$ ]]]]]]] $]^{14}$

Seen in this light, the analysis proposed in Section 4.2 exactly echoes an established parameter that assumes that, opposed to the case of languages such as English, there is a lack of remnant subject raising after Predicate Inversion in Chinese nominal phrases. As such, compared to Kayne's analysis, the line pursued in this paper could facilitate an explanation for cross-linguistically observed word order differences of a variety of nominal constructions via a unified parametric rule.

\section{Consequences and further discussion}

The present clausal analysis can help to explain the characteristics of the abstracttype [Num-MW-de-N] mentioned earlier. First, recall from (7) that de contained in the abstract-type [Num-MW-de-N] is obligatory, as repeated below:

(37) a. liang sheng *(de) rongji

two liter DE volume

'the volume of two liters'

b. wu mi $*(\mathrm{de})$ changdu

five meter DE length

'the length of five meters'

Under the present analysis, this can be explained in that de is an element popping up in the derivation of the abstract-type measurement construction for the sake of satisfying a well-formedness condition on Predicate Inversion, which requires that the functional head whose specifier hosts the inverted predicate be overtly realized. Given that along this line de is motivated entirely for structural purposes, it follows naturally that the existence of $d e$ is crucial to the grammaticality of the Chinese abstract-type measurement construction.

Second, recall that the head noun of the abstract-type [Num-MW-de-N] cannot be elided, with relevant examples reproduced below ((8b) repeated):

(38) wo xiang mai rongji da yidian de shuihu,

I want buy volume big a.little DE pot

zuihao neng you liang sheng de *(rongji).

better can have two liter DE volume

Intended: 'I want to buy a pot / pots with a little larger volume, which better can reach the volume of two liters.'

14 Similar to the cases of (30), the label FP represents the functional projection whose specifier position is the landing site of the raised remnant subject. The head complex LINKER+RELATOR, which is formed to license the predicate (i.e. the possessor Jean) to move across [Spec, RP] into [Spec, LP], has to raise to F as shown in (36b) to render [Spec, FP] and [Spec, LP] equidistant for voiture in [Spec, RP] to move into. 
To explain this, it is worth indicting that in accordance with the standard Predicate Inversion theory, the movement of RELATOR to LINKER has an effect of creating a new, extended phase (established based on the RP), i.e. the LP (cf. den Dikken 2006, 2007). This gives rise to a scenario that upon completion of Predicate Inversion, the underlying subject at $[\mathrm{Spec}, \mathrm{RP}]$ will end up being "trapped" within the domain of the extended phase. As a result, the subject will not be able to participate in later syntactic operations. To exemplify such a syntactic inertness of the $\mathrm{N}$ in the abstract-type [Num-MW-de-N], observe that the $\mathrm{N}$ cannot participate in topicalization, as shown below:

(39) a. *rongji $i_{i}$, liang sheng de $t_{i}$ hen da. volume two liter $\mathrm{DE}$ very big Intended: 'As for volume, two liters is very big.'

b. * shengao $_{\mathrm{i}}$, jiejie de nanpengyou you yi mi ba de $\mathrm{t}_{\mathrm{i}}$. height sister $\mathrm{DE}$ boyfriend have one meter eight $\mathrm{DE}$ Intended: 'As for height, my sister's boyfriend is 1.8 meters.'

Then, on the assumption that ellipsis is a deletion operation taking place in the course of syntactic derivation and needs to be formally licensed (Saito et al. 2008; Corver \& van Koppen 2009), the non-licensing of N-ellipsis in the abstract-type [Num-MW-de-N] can be explained in that the $\mathrm{N}$, being embedded within a phase, is no longer "visible" to syntactic licensing conditions on ellipsis and thus can by no means be grammatically elided.

Third, the present clausal analysis makes it possible to explain an interesting fact that the [Num-MW-de] sequence in the abstract-type [Num-MW-de-N] cannot be stacked with other de-marked modifiers to form parallel modification constructions, as exemplified below:

(40) a. *liang sheng de, gang ce chulai de rongji $(=(10 \mathrm{~b}))$
two liter DE just measure out DE volume
'the volume which is two liters and which was just measured out'
b. *180 jin de, hui yingxiang shenti jiankang de tizhong
180 catty DE will affect body health DE weight
'the weight which is 180 catties and which will affect people's health'

To understand this, recall from Section 2 that the abstract-type [Num-MW-de-N] behaves differently from Chinese de-marked modification constructions in a number of ways (see e.g. the contrast between (40b) and (12c)). This arguably indicates that the [Num-MW-de] sequence contained in the abstract-type measurement construction should be essentially differentiated from genuine adnominal de-marked modifiers. ${ }^{15}$ Given that the [Num-MW-de] sequence in the abstract-type measurement construction is not a modifying expression in the first

15 Due to space limitations, this paper does not go into the issue regarding the syntax of Chinese de-marked modification constructions yet leaves it for a thorough study in a separate work. 
place, it turns out totally unsurprising that it would be incompatible with the use of parallel modification.

Last, the definite nature of the abstract-type [Num-MW-de-N] can help to explain why this construction cannot be preceded by another quantifier (as shown in (9b)) or be used as a predicative nominal (as attested in (15)). As has been claimed above, the abstract-type [Num-MW-de-N] is used to refer and underlyingly correlates with a definite DP structure. That being the case, the incapability of the abstract-type measurement construction to be quantized by a quantifier or to be used predicatively can be naturally accounted for with the aid of the standard assumptions in generative linguistics that quantifiers can only apply to NPs but not DPs, and that a predicative nominal needs to be property-denoting in nature.

Before concluding this paper, a few more words are needed on the use of [Num-MW-de-N] headed by a dimension-denoting noun. One reviewer raises an intriguing example as shown below, where [Num-MW-de-N] headed by a dimension-denoting noun exhibits an indefinite reading and allows for N-ellipsis:

(41) san mi de gaodu bi liang mi de (gaodu) shufu.

three meter DE height compare two meter DE height comfortable

'A height of three meters makes one more comfortable than one of two meters.'

To account for (41), it is important to notice that the predicate involved here is shufu 'comfortable', which is semantically about sensational feelings. Given that conceptually an abstract dimension category in itself would not be able to bring about any effects of comforting (or discomforting), the present paper claims that for the head noun gaodu 'height' and the predicate shufu 'comfortable' to be semantically compatible, gaodu needs to be "substantivized", namely, being conceived as denoting an entity perceptible to people. As such, in terms of interpretation, (41) is in fact similar to (42) as shown below, where the head noun of the nominal phrase is the entity-denoting noun shafa 'sofa'. Concretely, for (42), the measure phrases san mi and liang mi serve as modifiers of the entity-denoting noun 'sofa', with the whole [Num-MW-de-N] expression standing as a modifyingtype measurement construction. As expected, the head noun 'sofa' can be licitly elided, which is one of the characteristic properties owned by the modifying-type [Num-MW-de-N] (cf. Section 2). Also, as indicated by the English translation, both san mi de shafa and liang mi de (shafa) are not subject to the definiteness effect and can be used to mean indefinite referents.

(42) san mi de shafa bi liang mi de (shafa) shufu. three meter DE sofa compare two meter DE sofa comfortable 'A sofa of three meters (long) makes one more comfortable than one of two meters.'

With this in mind, the present paper proposes that the behaviors exhibited by san/ liang mi de gaodu in (41) can be understood from the perspective of its parallelism 
with san/liang mi de shafa in (42). That is, given that the meaning of gaodu in (41) is contextually "substantivized" and thus semantically akin to an entity-denoting noun, the corresponding san/liang mi de gaodu should fall under the same type of san/liang mi de shafa, i.e., the modifying-type [Num-MW-de-N]. Seen in this light, it follows naturally that the former would pattern with the latter both semantically and syntactically, such as licensing an indefinite interpretation and permitting head noun ellipsis.

This being the case though, it is worth emphasizing that what (41) shows is a scenario where an inherently dimension-denoting noun contextually obtains the use of an entity-denoting noun, as a result of which the corresponding [Num$\mathrm{MW}-d e-\mathrm{N}]$ can be analyzed as a modifying-type measurement construction. Nevertheless, it does not mean that there is no need to distinguish the abstracttype from the modifying-type [Num-MW-de-N]. As has been elaborated in Section 2 , for the cases where an entity-related interpretation is not contextually coerced for the dimension-denoting noun, a series of noticeable asymmetries are detected between the [Num-MW-de-N] construction headed by a dimension-denoting noun and that headed by an entity-denoting noun. This offers a strong indication that it is still necessary to fundamentally differentiate the abstract-type from the modifyingtype measurement construction; otherwise, the observed distinctions would turn out unexplainable. ${ }^{16}$

\section{Conclusion}

This paper looks into a special sub-type of measurement construction in Mandarin Chinese, i.e. [Num-MW-de-N] where the $\mathrm{N}$ contained is an abstract dimensiondenoting noun. It was first shown that the abstract-type [Num-MW-de-N] fundamentally differs from the quantifying-/modifying-type [Num-MW-de-N] in both syntax and semantics. Then it was illustrated that while [Num-MW] is a property-denoting expression, the abstract-type [Num-MW-de-N] is semantically definite, which is used to identify a particular measure value (denoted by [Num$\mathrm{MW}]$ ) along a well-defined dimension category (expressed by the $\mathrm{N}$ ).

At the syntactic level, a clausal approach was pursued to account for the derivation of the abstract-type [Num-MW-de-N]. Upon the claim that the dimension-denoting $\mathrm{N}$ and [Num-MW] entertain a direct predication relationship, it was hypothesized that the two start out forming a small clause in the underlying structure, with the $\mathrm{N}$ as the subject and [Num-MW] as the predicate. The surface word order of [Num-MW-de-N] is derived via the syntactic operation of Predicate Inversion, and the intervening $d e$ is the spell-out of the functional head whose specifier hosts the inverted predicate. Last, a parameter regarding the (non-)

16 I thank the reviewer very much for calling my attention to the example such as (41), which greatly helps to complete the current discussion on [Num-MW-de-N] headed by a dimension-denoting noun. 
application of remnant subject movement after Predicate Inversion was attempted to account for the word order distinction between Chinese and English abstracttype measurement constructions. The present analysis can not only capture the characteristics of the abstract-type [Num-MW-de-N] in Chinese but also facilitate a unified explanation for word order distinctions of different languages in a variety of nominal constructions.

\section{Acknowledgements}

Part of this article has been presented at the Symposium on Word Order in Chinese (The Chinese University of Hong Kong, May 2012) and the Symposium on Chinese Language Research and Teaching (The Education University of Hong Kong, May 2015). I am grateful for the comments from the audience. I also sincerely thank the three anonymous reviewers for their very helpful suggestions. All the remaining errors are my own.

\section{References}

Alexiadou, Artemis, Liliane Haegeman \& Melita Stavrou. 2007. Noun phrase in the generative perspective. Berlin/New York: Walter de Gruyter.

Bennis, Hans, Norbert Corver \& Marcel den Dikken. 1998. Predication in nominal phrases. Journal of Comparative Germanic Linguistics 1(2). 85-117.

Chierchia, Gennaro. 2005. Definites, locality, and intentional identity. In Gregory N. Carlson \& Francis Jeffry Pelletier (eds.), Reference and quantification: The Partee effect, 143-177. Stanford: CSLI.

Chomsky, Noam. 2000. Minimalist inquiries: The framework. In Roger Martin, David Michaels \& Juan Uriagereka (eds.), Step by step: Essays on minimalist syntax in honor of Howard Lasnik, 89-155. Cambridge, MA: MIT Press.

Corver, Norbert \& Marjo van Koppen. 2009. Let's focus on noun phrase ellipsis. Groninger Arbeiten zur Germanistischen Linguistik 48. 3-26.

Den Dikken, Marcel. 2006. Relators and linkers. Cambridge, MA: MIT Press.

Den Dikken, Marcel. 2007. Amharic relatives and possessives: Definiteness, agreement, and the linker. Linguistic Inquiry 38(2). 302-320.

Den Dikken, Marcel \& Pornsiri Singhapreecha. 2004. Complex noun phrases and linkers. Syntax 7(1). 1-54.

Dimitrova-Vulchanova, Mila \& Giuliana Giusti. 1998. Fragments of Balkan nominal structure. In Artemis Alexiadou \& Chris Wilder (eds.), Possessors, predicates and movement in the determiner phrase, 333-360. Amsterdam: John Benjamins.

Her, One-Soon \& Chen-Tien Hsieh. 2010. On the semantic distinction between classifiers and measure words in Chinese. Language and Linguistics 11(3). 527551.

Hsieh, Miao-Ling. 2008. The internal structure of noun phrases in Chinese. Taipei: Crane.

Jiang, Li Julie. 2008. Monotonicity and measure phrases in Chinese. Paper presented at the $11^{\text {th }}$ International Symposium on Chinese Languages and Linguistics, 
National Chiao Tung University, Taiwan, 23-25 May.

Jin, Jing. 2013. Functions of Chinese classifiers: A syntax-semantics interface account. Hong Kong: The Hong Kong Polytechnic University dissertation.

Jin, Jing. 2015. The partitive construction in Mandarin Chinese. International Journal of Chinese Linguistics 2(1). 85-120.

Jin, Jing. 2016. Hanyu [duliang duanyu+de+mingci] de zai fenlei [A subcategorization of [Measure Phrase+de+Noun] in Mandarin Chinese]. Dangdai Yuyanxue [Contemporary Linguistics] 18(2). 216-232.

Kayne, Richard S. 2003. Silent years, silent hours. In Lars-Olof Delsing, Josefsson Gunlög, Halldor Armann Sigurðsson \& Cecilia Falk (eds.), Grammar in focus: Festschrift for Christer Platzack, volume 2, 209-226. Lund: Wallin \& Dalholm.

Li, Xuping. 2011. On the semantics of classifiers in Chinese. Ramat Gan: Bar-Ilan University dissertation.

Li, Yen-Hui Audrey. 2013. P-insertion and ellipsis. Studies in Chinese Linguistics 34(2). 99-128.

Longobardi, Giuseppe. 1994. Reference and proper names: A theory of N-movement in syntax and logical form. Linguistic Inquiry 25(4). 609-665.

Moltmann, Friederike. 2009. Degree structure as trope structure: A trope-based analysis of positive and comparative adjectives. Linguistics and Philosophy 32(1). 51-94.

Saito, Mamoru, Tzong-Hong Jonah Lin \& Keiko Murasugi. 2008. N'-ellipsis and the structure of noun phrases in Chinese and Japanese. Journal of East Asian Linguistics 17(3). 247-271.

Schwarzschild, Roger. 2005. Measure phrases as modifiers of adjectives. Recherches linguistiques de Vincennes (34). 207-228.

Zamparelli, Roberto. 2000. Layers in the determiner phrase. New York: Garland.

Zhang, Ning Niina. 2013. Classifier structures in Mandarin Chinese (Trends in Linguistics. Studies and Monographs, Vol. 263). Berlin: De Gruyter Mouton.

Mailing Address: Department of Chinese Language Studies, The Education University of Hong Kong, 10 Lo Ping Road, Tai Po, New Territories, Hong Kong

Email: $\quad$ jjin@eduhk.hk

Received: $\quad$ September 3, 2015

Accepted: $\quad$ June 23, 2016 


\title{
論漢語抽象類度量短語的句法結構
}

\author{
金晶 \\ 香港教育大學
}

\section{提要}

本文討論漢語中一類特殊的度量短語, 即所含名詞表示抽象度量範疇的 [ 數詞 - 度 量詞 - “的” - 名詞 ]。抽象類度量短語與所含 [ 數詞 - 度量詞 ] 用於表示量化或修 飾具體事物名詞的 [ 數詞 - 度量詞 - “的” - 名詞 ] 有著本質上的不同。本文提出, 抽象類 [ 數詞 - 度量詞 - “的” - 名詞 ] 從語義上說是一個有定表達。在句法上, 該結構的衍生過程可通過謂語倒裝理論下的小句分析來解釋。最後, 本文從參數差 異角度探討了漢語抽象類度量短語中名詞居後、而英語抽象類度量短語中名詞居前 的原因, 提出這與謂語倒裝完成後是否還發生名詞提升有關。

\section{關鍵詞}

度量短語, 謂語倒裝, 語序, 漢語句法 